\title{
MUTATION SYSTEMS IN CULTURED MAMMALIAN CELLS*
}

\author{
Ernest H. Y. Chu \\ Department of Human Genetics \\ University of Michigan Medical School \\ Ann Arbor, Michigan 48109
}

In 1968 during the Thirteenth International Congress of Genetics in Tokyo, Heinrich Malling and I presented evidence for chemical induction of specific locus mutation in cultured mammalian cells. ${ }^{1,2}$ In the same meeting, Kao and Puck also reported the success of chemical mutagenesis in Chinese hamster cells. ${ }^{3,4}$ Following those initial experiments, there was a period of consolidation, mostly devoted to the demonstration that the heritable variations under study did include true mutations. Evidence for mutation has now been obtained in a great number of cell systems. This evidence has taken several forms, including quantitative predictions of the effects of ploidy, the efficacy of known mutagens in the induction of cell variants, and, most convincingly, the direct demonstration in mutant cells of altered gene products. ${ }^{5,6}$ Although we can take satisfaction from the conclusion that mutations can be experimentally induced and analyzed in cultured mammalian cells, certainly many important questions remain. In this presentation I shall attempt to provide the background on and introduce a series of questions, uncertainties, and investigative possibilities in this field.

The significant problems that persist in mammalian cell mutagenesis may be grouped into three overlapping areas of research:

1. The investigation of basic mutational events that take place in mammalian cells.

2. The development of methods for the quantitation of mutation induced in cultured mammalian cells.

3. The application of these methods for the screening of environmental mutagens.

For convenience of presentation, these areas will be covered in sequence.

\section{InVESTIGATION OF Basic Mutational Events}

Mutation is much more than a chemical or configurational change in DNA; it is a biological process deeply enmeshed in the structural and biological complexities of the cell and modulated by both internal and external factors. We still know little about the causes of spontaneous mutations, but the experimental evidence leaves no doubt that mutations occur in replicating as well as nonreplicating genomes of both pro- and eukaryotes. Our knowledge of the origin and nature of induced mutations far outstrips that relating to spontaneous ones, because it is always easier to analyze processes that one can manipulate experimentally than naturally occurring ones. Nevertheless, the manifestation of primary DNA lesion to a substantial alteration in gene expression follows a complex process, which acts as a sieve that allows only a proportion of the DNA lesion to proceed toward the final product of a mutant clone. According to

*Research supported by Public Health Service Grant CA 26803. 
Auerbach, the mutagenic process for induced mutations may include (1) penetration of the mutagen to DNA; (2) production of a premutational lesion; (3) death from unrepaired damage; (4) repair of lesion, restoring the normal gene; (5) fixation of the premutational lesion as a mutated gene; (6) formation of mutant cells; (7) death of mutant cells; and (8) formation of a clone of mutant cells. ${ }^{7}$ Two steps in the mutagenic process, namely, the premutational lesion caused by mispairing and the misrepair of the lesion, are the most important and basic mutational mechanisms. Many of the enzymes of DNA replication and repair contribute to the fate of primary DNA lesions, and the specificity of these enzymes contributes to the diversity of the mutational response.

Mutational responses in cultured mammalian cells can be measured at the DNA, protein, and phenotype levels. The entire way in which we think about genetic events, especially mutation, in mammalian cells may soon take on new meaning as the significance of introns and exons and genomic fluidity begins to be understood. These problems of understanding some of the most fundamental aspects of the structural organization of eukaryotic genes are exciting ones, especially in the promise of new recombinant DNA and nucleic acid sequencing techniques to aid in solving them. Studies of mutations of mammalian cells at the DNA level, as measured by either restriction analysis or nucleotide sequencing, are just the beginning.

Mutation can also be identified and measured at the protein level. Methods for oneor two-dimensional gel electrophoresis have been developed that are potentially powerful tools for monitoring mutations both in human somatic cells, in vitro and in vivo, and in human populations. If a change in electrophoretic mobility or molecular weight of a protein is indicated, the protein or peptide may be isolated for determination of amino acid composition and sequence. A specific mutation at the gene level may thus be inferred.

The effect of spontaneous and induced mutations on the expression of hypoxanthine guanine phosphoribosyltransferase (HPRT) (resistance to the analogues of the purine bases) in cultured mammalian somatic cells has been studied extensively. Several investigators have reported abnormalities in the immunochemical, catalytic, and electrophoretic properties of the enzyme in mutagenized HPRT-deficient cell lines and their revertants (reviewed in Reference 8). Alterations in peptide maps of the enzyme have been detected in several variant cell lines. ${ }^{9-11}$ These studies provide evidence for the existence of a variety of undefined mutations in the structural gene for HPRT in cultured mammalian cells.

In man, an inherited deficiency of HPRT is associated with two distinct clinical syndromes: a virtually complete deficiency of HPRT activity has been described in patients with Lesch-Nyhan syndrome, while a partial enzyme deficiency is found in some male patients presenting with hyperuricemia and a severe form of gout. Human HPRT exists, in its native state, as a tetramer of identical subunits, coded by a single $\mathrm{X}$-linked gene locus. ${ }^{12}$ The complete amino acid sequence of HPRT from human erythrocytes has been defined. ${ }^{13}$ Each subunit of the enzyme is 217 amino acids long with a molecular weight equal to 24,470 . Jolly et al. recently reported the isolation and preliminary characterization of cloned cDNA sequences of the human HPRT gene. ${ }^{14}$ The amino acid sequence predicted from the nucleotide sequence of the CDNA is in complete agreement with that defined by protein sequencing except that the $\mathrm{NH}_{2}$ terminal methionine coded for by the initiator codon is absent in the mature enzyme.

More recently, the molecular basis for HPRT deficiency in two unrelated gout patients has been defined as single amino acid substitutions. ${ }^{15.16}$ One enzyme variant,

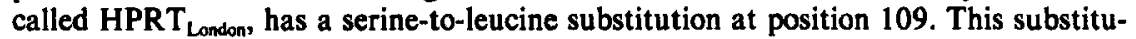
tion can be explained by a single nucleotide change in the codon for serine (UCA $\rightarrow$ 
UUA). Sequence analysis of a single peptide in the variant enzyme $\left(H P R T_{\text {Toronto }}\right)$ of another gout patient revealed an arginine-to-glycine amino acid substitution at position 50 (codon for $\mathrm{Arg}_{50}$ changed from CGA to GGA). These significant developments not only demonstrate for the first time the molecular basis of human mutation at the HPRT locus but also suggest the feasibility of identifying the molecular defects of HPRT mutants, spontaneous and induced, that can be obtained in cultured human cells.

The most prevailing end point for assessing mutations in cultured mammalian cells is a change in the cellular phenotype, such as nutritional requirement, resistance to cytotoxic agents, or cell membrane alterations. At this level, it is particularly important to distinguish the nature of somatic variations arising in vitro, whether it is genetic or epigenetic. Recently, Morgan Harris showed that brief exposure of 5-bromodeoxyuridine-tolerant, thymidine kinase-deficient $\left(\mathrm{TK}^{-}\right)$Chinese hamster cells to 5-azacytidine resulted in massive conversion to the $\mathrm{HAT}^{+}$state (ability to grow in medium containing hypoxanthine, aminopterin, and thymidine), ${ }^{17}$ suggesting that the induction of revertants might have resulted from changes in DNA methylation patterns. Similarly, 5-azacytidine treatment has been used to reactivate the expression of the HPRT gene on the inactive X chromosome in Chinese hamster cells. ${ }^{18}$

Even though the change is genetic, an alteration in the phenotype could be the result of either a genic or chromosomal event. For instance, Cox and Masson have shown that $\mathrm{x}$-ray-induced mutations to 6-thioguanine resistance in cultured human diploid fibroblasts consist mostly of chromosomal deletions or rearrangements. ${ }^{19}$ Hozier and his associates have made a cytogenetic analysis of the $\mathrm{L} 5178 \mathrm{Y} / \mathrm{TK}^{+/-} \rightarrow$ $\mathrm{TK}^{-1-}$ mouse lymphoma mutagenesis assay system. ${ }^{20}$ Mutant $\mathrm{TK}^{-1-}$ colonies form a bimodal frequency distribution of colony sizes for most mutagenic or carcinogenic test substances. Large-colony $\mathrm{TK}^{-/-}$mutants with normal growth kinetics appear karyologically identical within and among clones and with the $\mathrm{TK}^{+/-}$parental cells. In contrast, most slow-growing, small-colony $\mathrm{TK}^{-1-}$ mutants have a readily recognizable chromosome rearrangement involving the mouse chromosome 11 on which the TK locus is located.

Cell lines have been developed in which point mutation, chromosome loss, or chromosome deletion leading to the disappearance of a phenotype could be directly assayed. Puck has developed a test system in which the marker is contained on a chromosome not itself necessary for cell reproduction. ${ }^{21}$ One such example is a reduced human-Chinese hamster cell hybrid in which a single human chromosome 11 is retained and mutations at several loci on this chromosome, namely, the antigenic markers $a_{1}, a_{2}, a_{3}$, LDH-A, and AcP-2, can be induced with mutagens. Any possible modification of a human protein or enzyme and the presence or absence of chromosome 11 or its segment can be studied. Using two-dimensional gel electrophoresis, at least eight distinct spots unique to the cell containing chromosome 11 have been located on the electrophoretogram. ${ }^{22}$

\section{Development of Methods for Quantitation of Mutation induction in Cultured Mammalian Cells}

\section{Choice of Cell Material}

A general question regarding any study of cultured cells is whether it accurately reflects the cellular responses in the intact animal. This point may be addressed using the direct-acting mutagen testing systems in which peripheral lymphocytes in the 
intact animal are examined. Another question is, Can the results derived with rodent cells be extended to the cells of humans? There is evidence that the repair of DNA damage differs both in process and extent between human and rodent cells. ${ }^{23}$ Clearly, our knowledge of the similarities and differences in DNA replication and repair of human and other mammalian cells is lacking because of the dearth of human cell mutants for these fundamental processes. Therefore, studies of mutagenesis with human diploid fibroblasts, lymphoblastoid cell lines, or other established near-diploid cell lines should reduce the necessity for extrapolation to man.

\section{Development of Selectable Genetic Markers}

On the basis of classical studies with corn and Drosophila, the rates of spontaneous mutation may differ among different loci. Evidence has been presented that in certain mutator strains of Chinese hamster ovary (CHO) cells, the spontaneous mutation rates were 5- to 50-fold higher than those in parental cells for two genetic markers (resistance to 6-thioguanine or to ouabain), but the rates for two other markers (reversion of proline auxotrophy to prototrophy and forward mutation to emetine resistance) were unaffected. ${ }^{24}$ As shown by several investigators, certain agents such as $x$-rays and gamma-rays fail to induce ouabain-resistant mutants in mammalian cells, ${ }^{25-27}$ although they are capable of inducing 6-thioguanine-resistant mutants in the same cell type. Therefore, it appears that mutation rates may be site specific for mammalian cells as well. From both the theoretical and practical points of view, one of the important tasks is to develop additional selectable genetic markers for comparative mutagenesis among loci and for more accurate assessment of mutagenic potential of environmental chemicals.

\section{Metabolic Activation}

Environmental chemical mutagens often are nonreactive and have to be enzymatically activated before they can manifest their biological effects. Many cell types, including $\mathrm{CHO}$ and mouse lymphoma L5178Y cells, are not capable of activating procarcinogens or promutagens. Mutagenesis in these cells has to be studied by the addition of enzymatically active liver subcellular fraction, or by cocultivation with intact hepatocytes (cell-mediated mutagenesis). Tong and Williams have developed a system in which adult rat liver epithelial cells are used to detect genotoxic compounds by mutations at the HPRT locus. ${ }^{28}$

The subcellular enzyme fractions, such as rat liver microsomal S-9 fractions, are simpler to use and have been shown to activate a wide variety of procarcinogens. However, it has been shown that the profile of the metabolites and DNA adducts formed after metabolism of various potent carcinogens is different from that of intact cells. This indicates that the use of subcellular fractions does not accurately simulate the in vivo situation. It is also known that for certain classes of chemicals, metabolic activation or inactivation is species specific. If it can be metabolically activated in vitro, will it also be activated in humans? The major dilemma here is that given a chemical about which little is known, it is difficult to ensure that the activation system used will detect its possible mutagenic potential. Furthermore, the concentration of the S-9 fraction used in the cell-mutagenesis experiments may affect cell viability, thus introducing another source of biological variability to quantitative mutagenesis. 


\section{Phenotypic Expression}

The course of events from the initial mutagenic insult to a cell to the appearance of the cell's progeny as a mutant colony is complicated and largely undefined. The so-called phenotypic expression refers to this series of unknown processes leading to the eventual recognition of the mutant. Forward mutations, such as $\mathrm{HPRT}^{-}$or $\mathrm{TK}^{-}$, which represents a loss of function, could be the result of a spectrum of mutational events including chromosome loss, chromosomal deletion, nucleotide base substitution, and base addition or deletion (frameshift changes). The lesion, such as a loss or change of a base, could be repaired without error, thus producing no mutation, or could be misrepaired, leading to a change (mutation) in the normal nucleotide sequence. In theory, two rounds of cell replication are needed for "error replication" resulting in an altered base pair. Furthermore, cell divisions are necessary to allow dilution or degradation of the parental mRNA and/or enzyme in the cell carrying the mutated gene. These are the reasons why a period of phenotypic expression between the removal of the mutagen and the addition of the selective agent is necessary. The length of this period has been experimentally determined for a maximum recovery of the HPRT ${ }^{-}$ and $\mathrm{TK}^{-}$mutations, and different lengths of time have been shown for mutants at other loci.

For both test systems using $\mathrm{CHO}$ or $\mathrm{L} 5178 \mathrm{Y}$ cells, a predetermined period for phenotypic expression is allowed. Li devised a simple technique for growth of CHO cells as unattached cultures. ${ }^{29}$ The optimum expression time was shorter for unattached cells (six days) than for attached cells (nine days). However, it is conceivable that different newly arisen mutants may have different growth rates and different selective advantages, relative to each other and to the parental cells. Some mutants may have divided more frequently than the parental cells and are overrepresented; others may be very slow growers, which may be missed in an enumeration of mutant colonies at a fixed time. Still other mutants may not thrive at all. Using the respreading technique, the mutation frequency is therefore only an average estimate.

\section{Cell Density (Metabolic Cooperation)}

Another factor that may affect mutation frequency is cell density. It was shown, especially in the case of $\mathrm{HPRT}^{-}$mutant selection, that intercellular communication upon contact will diminish the number of mutants recovered. This phenomenon, known as "metabolic cooperation," occurs during 6-thioguanine selection and entails a transfer of metabolites, such as 6-thioguanosine monophosphate, through the gap junctions on the cell membrane from the dying parental cells to mutants, killing the latter in the process. For $\mathrm{CHO}$ cells, reconstruction experiments have determined the maximum cell density $\left(2 \times 10^{5} / 10 \mathrm{~cm}\right.$ dish) that would not affect $\mathrm{HPRT}^{-}$mutant frequency.

The problem of metabolic cooperation may be less serious in L5178Y cells grown in suspension due to a small number of gap junctions on the cell surface. Metabolic cooperation may also be reduced or abolished in the presence of tumor promoters (P. Liu, personal communication). For certain membrane markers, such as resistance to ouabain or abrin, ${ }^{30}$ there was no demonstrable metabolic cooperation. For still other genetic markers under different situations, cross feeding in crowded cultures may actually increase, rather than decrease, the mutation frequency. Harris showed that the incidence of spontaneous variation to puromycin resistance in pig kidney cells increased in proportion to population density. ${ }^{31}$ Large colonies or aggregates of 
sensitive cells grew progressively in puromycin at concentrations that destroyed the same populations completely when present in dispersed form. Harris concluded that response to puromycin is conditioned by cellular interactions as well as by genetic susceptibility.

\section{Back Mutation and Preexisting Mutants}

It is generally assumed that there is no back mutation during the course of mutagenicity testing, but this possibility needs to be verified. If it does indeed occur, the frequency should be accounted for in an estimate of the spontaneous and induced forward mutations.

The existence of preexisting mutants of spontaneous origin may be troublesome in the evaluation of a weak mutagen. There are experimental procedures, however, such as recloning or pregrowth in counterselective media, to eliminate or reduce the number of preexisting mutants in cell populations prior to mutagenicity testing.

\section{Effects at Low Doses}

At very low doses of chemicals, the linearity of dose-effect curves has not been settled. In both $\mathrm{CHO}$ and the mouse lymphoma cell systems, dose-effect relationships within a certain range of concentrations have been obtained for many chemicals, such as ethyl methanesulfonate and ethyl nitrosourea. However, it is debatable whether a linear extrapolation to lower doses is either reasonable or desirable.

A number of biological factors may play important roles during the entry of a chemical (or its derivatives) into the cell and the nucleus before its interaction with the genetic material. The question in a testing situation is to find the lowest effective dose that can induce mutation, not the theoretical threshold. The practical problem is the chronic exposure to low doses, rather than the demonstration of mutation induction after a single acute exposure at a very low dose. It seems that a dose-response curve using a series of doses is more informative than the determination of a "doubling dose" of the spontaneous frequency.

\section{Application of Mammalian Cell Mutation Assay Systems for the SCREENING OF ENVIRONMENTAL MUTAGENS}

\section{Choice of Test Systems}

Numerous cell lines, markers, and selective systems are now available. The use of human versus nonhuman somatic cells in cultures for mutation assay may be considered on the basis of technical advantages and relevance to man. The extent to which the assays could or should be used is a matter of controversy. Some people may feel that the assays are functional and the available systems should now be employed as far as practicable in the process of hazard evaluation. To wait for the ideal system is, in fact, a decision to continue to expose people and the environment to chemicals of unknown activity. At the Banbury conference in 1979, the weight of opinion was that the present test systems used in conjunction with various other end points are better than nothing. It was considered better to have a few false positives and negatives rather than exposure to a whole lot of potentially hazardous chemicals. 


\section{Definition of Basic Parameters of a Mutagen Screening System}

Many factors must be examined to distinguish between genetic and epigenetic events, and certain basic criteria must be met if one claims to have a true mutational system. The types of mutation that are measured, whether genic, chromosomal, or both, should be delineated. In fact, for screening of mutagenicity of environmental chemicals, forward mutations-which encompass a broad spectrum of mutational events-may be preferable to reverse mutations. On the other hand, reverse mutations can help to identify the mutagenic specificity of chemicals.

The fact that many have developed reliable mutation systems does not imply that the work is easy or without problems. Many problems must be addressed for each system. Laboratories adopting a system should standardize the assay conditions and demonstrate that consistent, reproducible results can be obtained. The use of positive and solvent controls, the use of appropriate activation systems, background mutation frequencies consistent with historical controls, dose-response curves, and other baseline information should be defined. It may also be helpful to establish the classes of carcinogens and mutagens that the system accurately detects.

\section{Increase in the Mutability of Cells}

Recent years have witnessed a sharply increased number of studies of mutator and antimutator mutations in a variety of organisms, the most penetrating analyses being conducted in prokaryotes and fungi. These studies have proceeded in parallel with analyses of mechanisms of DNA replication and repair, at the same time as genes responsible for these functions have been identified by conditional lethal and/or mutagen-sensitive mutations.

In mammalian cells, increased rates of spontaneous mutations have been demonstrated in mutator strains that have modified intracellular nucleoside triphosphate pools. $24,32,33$ In addition to cell strains obtained from the repair-defective human syndromes, several laboratories have reported the isolation of mutants in cultured rodent cells that have increased sensitivity to killing by mutagens, such as ultraviolet (UV) light (e.g., References 34-36). In some of these UV-sensitive mutants, enhancement of UV mutagenic response has been demonstrated. ${ }^{37}$ Conceivably, these and other hypermutable cell strains will be very useful in increasing the sensitivity for detecting certain classes of mutagens. It is of particular importance to obtain human cell mutants defective in DNA repair for such purposes.

\section{Statistical Analysis}

In quantitative measurements of cell survival and mutation frequency, there are a number of sources of errors that affect the final mutant yield, including cell counts, cell viability during cytotoxicity tests, growth rate, and competition among the newly arisen mutants. Appropriate statistical models to account for these sources of variability and the choice of statistical methods for the analysis of data are important considerations in screening tests of environmental mutagens and carcinogens. There seem to be many valid problems in this area that require further research and discussion. A workshop on statistical analysis of in vitro tests for mutagenicity was held in April 1981 in Chapel Hill, North Carolina, under the joint sponsorship of the National Institute of Environmental Health Sciences and the National Toxicology Program. A report of the workshop will be published. 


\section{SUMMARY}

Experimental studies on the complex process of mutagenesis are conducted in the belief that they will increase our understanding of the effects of environmental agents on human populations. Cultured mammalian cells offer distinct advantages over other biological systems for studying this problem. Mutagenesis in cultured mammalian cells is unquestionably more relevant to man than are bacterial, fungal, or insect systems. At the same time, compared to whole-animal studies, cultured cells offer the advantage of ease of handling, low cost, and rapidity of assay. ${ }^{38}$

Mutation in cultured mammalian cells can be identified and measured at the phenotypic, protein, and DNA levels. Test systems have been developed to detect both gene and chromosome mutations. Direct evidence is available for true mutations occurring in mammalian cells, although some of the heritable variations could be epigenetic. It is now possible to identify the specific molecular alternations in mammalian cell mutants arising in vitro.

The development of methods for the quantitation of mutation in cultured mammalian cells has been briefly considered in this presentation, particularly with reference to the choice of cell material, development of selective markers, and the various factors that affect quantitative measurement of mutations. Although notable progress has been made, much still remains to be learned to make these measurements more reliable.

In applying the mammalian cell mutation assays for the screening of environmental mutagens, the choice of a system or systems should be based on their technical advantages and relevance to man. The basic parameters of the mutagen screening system must be well defined and controlled. The sensitivity for detection may be increased by the use of hypermutable cell strains. Appropriate statistical models to account for various sources of variability and the choice of statistical methods for the analysis of data are important considerations in screening environmental mutagens and carcinogens.

\section{REFERENCES}

1. ChU, E. H. Y. \& H. V. Malling. 1968. Chemical mutagenesis in Chinese hamster cells in vitro. In Proceedings of the 12th International Congress on Genetics. 1: 102. Science Council of Japan. Tokyo, Japan.

2. CHU, E. H. Y. \& H. V. MALling. 1968. Mammalian cell genetics. II. Chemical induction of specific locus mutations in Chinese hamster cells in vitro. Proc. Nat. Acad. Sci. USA 61: $1306-1312$.

3. KAO, F.-T. \& T. T. PuCK. 1968. Isolation of nutritionally deficient mutants of Chinese hamster cells. In Proceedings of the 12th International Congress on Genetics. 1: 157. Science Council of Japan. Tokyo, Japan.

4. KAO, F.-T. \& T. T. PUCK. 1968. Genetics of somatic mammalian cells. VII. Induction and isolation of nutritional mutants in Chinese hamster cells. Proc. Nat. Acad. Sci. USA 60: $1275-1281$.

5. CHU, E. H. Y. 1974. Induction and analysis of gene mutations in cultured mammalian somatic cells. Genetics 78: 115-132.

6. SiminovitCH, L. 1976. On the nature of heritable variation in cultured somatic cells. Cell 7: 1-11.

7. Auerbach, C. 1976. Mutation Research. Problems, Results and Perspectives: 381-388. Chapman and Hall. London, England.

8. Caskey, C. T. \& G. D. Kruh. 1979. The HPRT locus. Cell 16: 1-9.

9. Capecchi, M. R., R. A. Von der HaAR, N. E. CAPecchi \& M. M. Sveda. 1977. The isolation of a suppressible nonsense mutant in mammalian cells. Cell 12: 371-381. 
10. Milman, G., S. W. Krauss \& A. S. OlSEN. 1977. Tryptic peptide analysis of normal and mutant forms of hypoxanthine phosphoribosyltransferase from HeLa cells. Proc. Nat. Acad. Sci. USA 74: 926-930.

11. KRUh, C. D., R. G. Fenwick, JR. \& C. T. CASKey. 1981. Structural analysis of mutant and revertant forms of Chinese hamster hypoxanthine-guanine phosphoribosyltransferase. J. Biol. Chem. 256: 2878-2886.

12. Holden, J. A. \& W. N. Kelley. 1978. Human hypoxanthine-guanine phosphoribosyltransferase: evidence for a tetrameric structure. J. Biol. Chem. 253: 4459-4463.

13. Wilson, J. M., G. E. Tark, W. C. Mahoney \& W. N. Kelley. 1982. Human hypoxanthine-guanine phosphoribosyltransferase: complete amino acid sequence of the erythrocyte enzyme. J. Biol. Chem. 257: 10978-10985.

14. Jolly, D. J., A. C. Esty, H. U. Bernard \& T. Friedmann. 1982. Isolation of a genomic clone partially encoding human hypoxanthine phosphoribosyltransferase. Proc. Nat. Acad. Sci. USA 79: 5038-5041.

15. Wilson, J. M., G. E. TARr \& W. N. Kelley. 1983. Human hypoxanthine-guanine phosphoribosyltransferase: a single amino acid substitution in a mutant form of the enzyme isolated from a patient with gout. Proc. Nat. Acad. Sci. USA. (In press.)

16. Wilson, J. M., I. H. Fox, G. E. TARR \& W. N. Kelley. The molecular abnormality in a structural variant of hypoxanthine-guanine phosphoribosyltransferase (HPRT Toronto $_{\text {) }}$ isolated from a patient with gout. J. Biol. Chem. (Submitted.)

17. HARRIS, M. 1982. Induction of thymidine kinase in enzyme-deficient Chinese hamster cells. Cell 29: 483-492.

18. GRANT, S. G. \& R. G. WORTON. 1982. 5-Azacytidine-induced reactivation of HPRT on the inactive $X$ chromosome in diploid Chinese hamster cells. Am. J. Hum. Genet. 34: $171 \mathrm{~A}$.

19. Cox, R. \& W. K. MASSON. 1978. Do radiation-induced thioguanine-resistant mutants of cultured mammalian cells arise by HGPRT gene mutation or X-chromosome rearrangement? Nature 276: 629-630.

20. Hozier, J., J. Sawyer, M. Moore, B. Howard \& D. Clive. 1981. Cytogenetic analysis of the $\mathrm{L}^{5} 178 / \mathrm{TK}^{+/-} \rightarrow \mathrm{TK}^{-1-}$ mouse lymphoma mutagenesis assay system. Mutat. Res. 84: $169-181$.

21. PUCK, T. T. 1979. Historical perspective on mutation studies with somatic mammalian cells. In Mammalian Cell Mutagenesis: The Maturation of Test System(s). Banbury Report 2. A. W. Hsie, J. P. O'Neill \& V. K. McElheny, Eds.: 3-14. Cold Spring Harbor Laboratory. Cold Spring Harbor, N.Y.

22. Scoggin, C. H., E. Gabrielson, J. N. Davidson, C. Jones, D. Patterson \& T. T. Puck. 1981. Two dimensional electrophoresis of human-CHO cell hybrids containing human chromosome 11. Somat. Cell Genet. 7: 389-398.

23. Regan, J. D. \& R. B. Setlow. 1973. Repair of chemical damage to human DNA. In Chemical Mutagens: Principles and Methods for Their Detection. A. Hollaender, Ed. 3: 151-170. Plenum Press. New York, N.Y.

24. Meuth, M., N. L'HeureuX-Huard \& M. Trudel. 1979. Characterization of a mutator gene in Chinese hamster ovary cells. Proc. Nat. Acad. Sci. USA 76: 6505-6509.

25. Arlett, C. F., D. Turnbull, S. A. Harcourt, A. R. Lehmann \& C. M. Colella. 1975. A comparison of the 8-azaguanine and ouabain-resistance systems for the selection of induced mutant Chinese hamster cells. Mutat. Res. 33: 261-278.

26. Chang, C. C., J. E. Trosko \& T. Akera. 1978. Characterization of ultraviolet lightinduced ouabain-resistant mutations in Chinese hamster cells. Mutat. Res. 51: 85-98.

27. Thacker, J., M. A. Stephens \& A. Stretch. 1978. Mutation to ouabain-resistance in Chinese hamster cells: induction by ethyl methanesulfonate and lack of induction by ionizing radiation. Mutat. Res. 51: 255-270.

28. Tong, C. \& G. M. Williams. 1980. Definition of conditions for the detection of genotoxic chemicals in the adult rat-liver epithelial cell/hypoxanthine-guanine phosphoribosyl transferase (ARL/HGPRT) mutagenesis assay. Mutat. Res. 74: 19.

29. LI, A. P. 1981. Simplification on the CHO/HGPRT mutation assay through the growth of Chinese hamster ovary cells as unattached cultures. Mutat. Res. 85: 165-175.

30. LI, 1.-C., D. A. Blake, I. J. Goldstein \& E. H. Y. ChU. 1980. Modification of cell membrane in variants of Chinese hamster cells resistant to abrin. Exp. Cell Res. 129: $351-360$. 
31. Harris, M. 1967. Phenotypic expression of drug resistance in cell culture. J. Nat. Cancer Inst. 38: 185-192.

32. Chang, C.-C., J. A. Boezi, S. T. Warren, C. L. K. Sabourin, P. K. Liu, L. Glatzer \& J. E. Trosko. 1981. Isolation and characterization of a UV-sensitive hypermutable aphidicolin-resistant Chinese hamster cell line. Somat. Cell Genet. 7: 235-253.

33. Weinaurg, G., B. Ullman \& D. W. Martin, JR. 1981. Mutator phenotypes in mammalian cell mutants with distinct biochemical defects and abnormal deoxyribonucleoside triphosphate pools. Proc. Nat. Acad. Sci. USA 78: 2447-2451.

34. KUROKI, T. \& S. Y. MIYAShiTA. 1976. Isolation of UV-sensitive clones from mouse cell lines by Lederberg style replica plating. J. Cell. Physiol. 90: 79-90.

35. Shiomi, T. \& K. SATo. 1979. Isolation of UV-sensitive variants of human FL cells by a viral suicide method. Somat. Cell Genet. 5: 193-201.

36. Thompson, L. H., J. S. Rubin, J. E. Cleaver, G. F. Whitmore \& K. Brookman. 1980. A screening method for isolating DNA repair-deficient mutants of $\mathrm{CHO}$ cells. Somat. Cell Genet. 6: 391-405.

37. Busch, D. B., J. E. Cleaver \& D. A. Glaser. 1980. Large scale isolation of UV-sensitive clones of $\mathrm{CHO}$ cells. Somat. Cell Genet. 6: 407-418.

38. Cox, R. 1982. Mechanisms of mutagenesis in cultured mammalian cells. In Environmental Mutagens and Carcinogens. T. Sugimura, S. Kondo \& H. Takebe, Eds.: 157-166. A. R. Liss, Inc. New York, N.Y. 